\title{
Design and Analysis of an Origami-Inspired Deployable Metamorphic Robotic Grasper
}

\author{
Sen Wang ${ }^{1,2}$, Jun $\mathrm{Xia}^{1,2}$, Hailin Huang ${ }^{1,2}$, Bing $\mathrm{Li}^{1,2^{*}}$, Ying $\mathrm{Hu}^{3 *}$ and Liu Rongqiang ${ }^{1}$ \\ ${ }^{1}$ State Key Laboratory of Robotics and System, Harbin Institute of Technology, Harbin 150001, P.R. China \\ ${ }^{2}$ Department of Mechanical Engineering and Automation, Harbin Institute of Technology, Shenzhen 518055, P.R. China \\ ${ }^{3}$ Shenzhen Institutes of Advanced Technology, Chinese Academy of Sciences, Shenzhen, 518055, P.R. China \\ * libing.sgs@hit.edu.cn and ying.hu@siat.ac.cn
}

\begin{abstract}
In this paper, an origami-inspired deployable metamorphic robotic grasper (ODMRG) with three limbs is presented, which can be used to grasp the large-scale unknown objects. First, using thickness-accommodation technique, a thick-origami basic module is introduced, and its mobility is also calculated by applying screw theory. The single limb consisting of four motion modules and one fixed module is illustrated. There are two metamorphic positions that enable this limb to have four different configurations from the stored state to grasping state. The metamorphic process and mobility analysis of such limb are provided in this paper. Then, the assembly of 3-limbs grasper is described, after which the grasper can be stored tightly and deployed compactly. In addition, the discussion about deploy/fold ratio of this grasper is given, which indicates that the deploy/fold ratio of this grasper can be increased by adding the number of modules. Finally, the workspace and dexterity of the grasper is presented. The shape adaptability in grasping are also tested, which shows that the gasper can adapt to the large-scale objects in different shapes.
\end{abstract}

Keywords-deployable robotic grasper, origami-inspired mechanism, metamorphic mechanism, variable deploy/fold ratio, shape adaptability.

\section{INTRODUCTION}

In recent years, the research on robotic grasper has become a hot topic, various rigid and flexible graspers have been proposed [1]. And with the deepening of research, robotic graspers are not only widely used in industry to complete the simple handing functions, they are also used in space engineering [2]. In particularly NASA demonstrated that there are many space junks in space that should be grasped and clearup to avoid them doing harm to operational spacecrafts [3]. However, it is a challenging work to grasp the space junks since the grasping objects are non-cooperative in such a space unstructured environment [4]. There are some uncertain factors for the objects, such as shape, size, location, velocity and so on, which require the robotic grasper to be large enough, and have good shape adaptability and dexterity. In addition, limited by

This work is financially supported in part by the National Natural Science Foundation of China [Grant Nos. 51835002 and U1613201], in part by the Shenzhen Research Funds [Grant Nos. JCYJ20170413104438332 and JCYJ20170811160940162]. the costs of manufacturing and transportation, the robotic grasper should be lightweight and deployable, and the deploy/fold ratio of the grasper is as large as possible, which means that the grasper should be folded tightly when stored and deployed completely when grasping.

There are three types of mechanical scheme in grasper design, serial mechanisms, parallel mechanisms, and serialparallel mechanisms. The traditional serial mechanisms have a large workspace, but the folding performances of them are poor and the number of actuators increases as the number of joints increases [5]. The grasper is designed by parallel mechanisms with higher carrying capacity and stiffness, but its workspace is limited [6]. In contrast, serial-parallel mechanisms combine the advantages of the former two mechanisms, which not only have large workspace, but also ensure the stiffness of robotic grasper [7][8]. Based on this scheme, Li proposed a novel deployable manipulator to grasp the large-scale unknown objects [9], Jia presented a systematic approach for the metamorphic mechanism modules synthesis, and these modules were used to construct a novel deployable grasping manipulator [10]. However, although these novel mechanisms can be deployed, the deploy/fold ratio of them are limited and constant, and if the number of modules in each grasping manipulator is increased to grasp the larger objects, their folding volume will still be large.

Origami as a traditional art of paper folding, wherein twodimensional thin-panel materials such as paper are folded into three-dimensional objects. Origami-inspired design can enable a foldable structure to be lightweight, compact and scalable while maintaining kinematic behavior [11], one of its greatest advantages is that it has a large deploy/fold ratio [12]. Based on these features, origami has been widely studied and applied in engineering fields [13][14]. In order to design a grasper with large deploy/fold ratio that suitable for space engineering, inspired by origami, this paper presents a 3-limbs origamiinspired deployable metamorphic robotic grasper (ODMRG), which can be stored tightly and deployed completely to grasp the large-scale unknown objects. In each origami-inspired basic module, there is only one motion can be realized. When the several basic modules are connected to form a single limb, 
there are two metamorphic positions that enables this limb to have four different configurations from the stored state to grasping state, and the deploy/fold ratio of this grasper will increase as the number of modules increases in each limb. The mobility, deploy/fold ratio, workspace, and dexterity are analyzed. Meanwhile, the prototype of 3-limbs ODMRG, which has four motion modules and a fixed module in each limb, is manufactured, and the shape adaptability is tested to prove the excellent grasping performance.

\section{Proposal of The 3-Limb DePloyable MetamorPhiC ROBOTIC GRASPER}

\section{A. Design of the Metamorphic Single Limb}

It is a common origami that two bigger congruent rectangles are used as the two side faces and two smaller congruent rectangles are combined to form the third side face of the triangular prism, such as the folding method of desk calendar. As shown in Fig. 1, if we ignore the thickness of paper, it can be folded and deployed completely. In folded state, different modules can be rotated around the creases in state 1. When all modules are in the same plane as state 2 , they can be deployed completely to state 3. Meanwhile, if we cut the creases between the larger rectangular facets, as shown in Fig. 1(b), these modules can be rotated around the creases between smaller rectangular facets in adjacent modules as state 4 .

Inspired by this type of origami, we can design an OMDGR, and the four states of origami also can be used to describe the grasper, including stored state, first deployed state, grasping facet deployed state and grasping state, which can be named from state 1 to state 4 . After adding the thickness for each facet, the basic origami-inspired module is as shown in Fig. 2. In this paper, $\varphi_{i j}$ represents the $j$ th facet in module $i$, and $\mathrm{R}_{i j}$ represents the $j$ th revolute joint in module $i$. In this basic module, two support facets $\varphi_{11}$ and $\varphi_{12}$ are connected by $\mathrm{R}_{11}$, two grasping facets $\varphi_{13}$ and $\varphi_{14}$ are connected by $\mathrm{R}_{12}$, $\varphi_{11}$ is connected with $\varphi_{13}$ by revolute joint $\mathrm{R}_{13}$, and $\varphi_{12}$ is connected with $\varphi_{14}$ by revolute joint $\mathrm{R}_{14}$.

To realize the change in the mobility from folded state to grasping state, the module must realize the grasping facet deployment motion. A coordinate frame $\{O-X Y Z\}$ is set at the position that intersection point between axis of $\mathrm{R}_{11}$ and the side face of $\varphi_{11}$, and the directions of three coordinate axes is

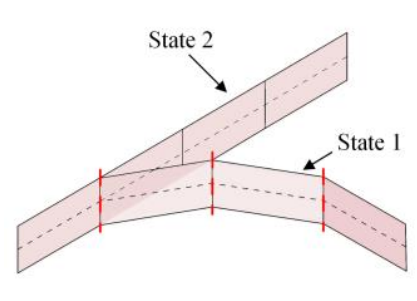

(a) The folded state

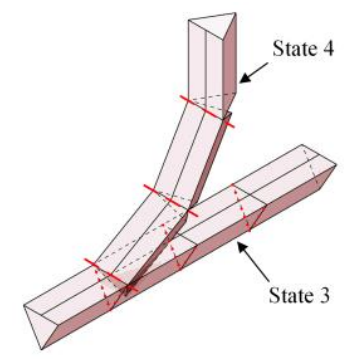

(b) The deployed state
Figure 1. Different states of origami

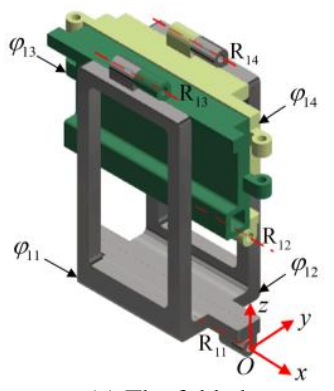

(a) The folded state

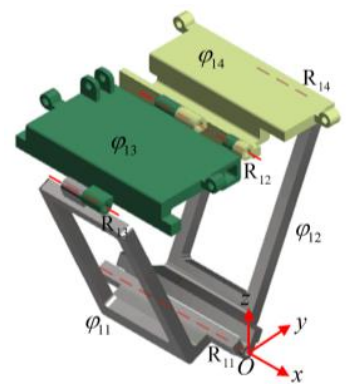

(b) The deployed state
Figure 2. Basic origami-inspired module

shown in Fig. 2. Since the four revolute joints are parallel to each other, this module is actually a planar four-revolute (4R) mechanism, and the twist system of this module is shown as:

$$
\left\{\begin{array}{l}
\boldsymbol{S}_{1}=\left(\begin{array}{lllll}
1 & 0 & 0 ; 0 & 0 & 0
\end{array}\right) \\
\boldsymbol{S}_{2}=\left(\begin{array}{lllll}
1 & 0 & 0 ; 0 & \mathrm{z}_{2} & 0
\end{array}\right) \\
\boldsymbol{S}_{3}=\left(\begin{array}{lllll}
1 & 0 & 0 ; 0 & \mathrm{z}_{3} & -y_{3}
\end{array}\right) \\
\boldsymbol{S}_{4}=\left(\begin{array}{lllll}
1 & 0 & 0 ; 0 & \mathrm{z}_{4} & -y_{4}
\end{array}\right)
\end{array}\right.
$$

The corresponding reciprocal screw system can be given as:

$$
\left\{\begin{array}{l}
\boldsymbol{S}_{1}^{r}=\left(\begin{array}{lllll}
1 & 0 & 0 ; 0 & 0 & 0
\end{array}\right) \\
\boldsymbol{S}_{2}^{r}=\left(\begin{array}{lllll}
0 & 0 & 0 ; 0 & 1 & 0
\end{array}\right) \\
\boldsymbol{S}_{3}^{r}=\left(\begin{array}{lllll}
0 & 0 & 0 ; 0 & 0 & 1
\end{array}\right)
\end{array}\right.
$$

Based on the Modified Gübler-Kutzbach formula:

$$
M=d(n-g-1)+\sum_{i=1}^{g} f_{i}+v-\xi
$$

where $d$ is the order of the mechanism, according to the common constraints (2), $d=6-3=3 ; n$ denotes the number of links and $n=4 ; g$ presents the number of joints, i.e., $g=4$; the degree of freedom for $i$ joint $f_{i}$ is only one; and there are not redundant constraint $v$ and local mobility $\xi$. Thus, the mobility of this module is only one.

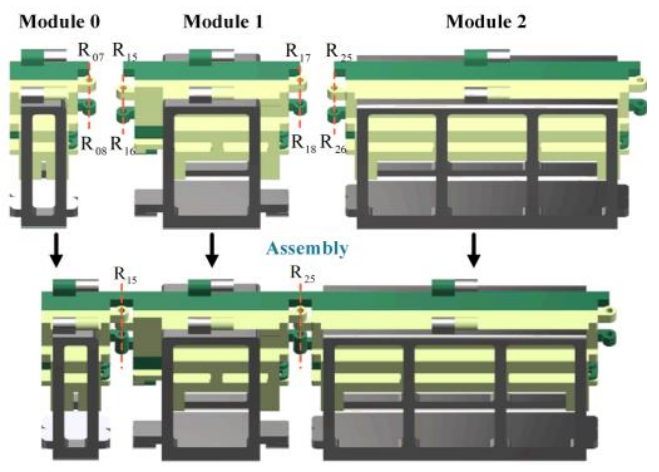

Figure 3. The assembly of adjacent modules 


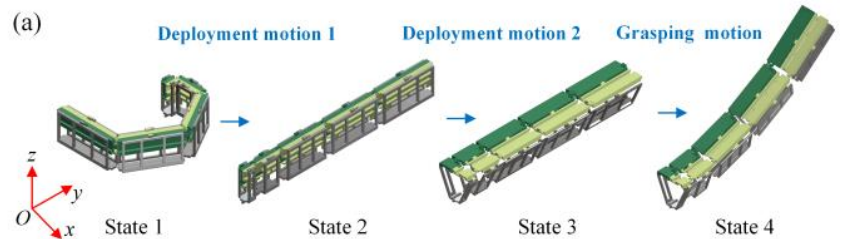

(b)

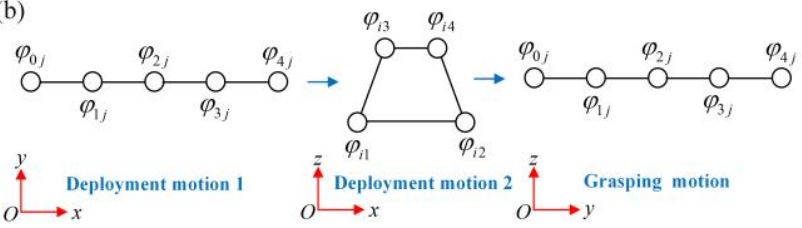

Figure 4. The motions of a single limb and the corresponding topologies

In order to obtain the single limb of grasper, we should design the assembly method of each adjacent module. It is different with traditional graspers that ODMRG needs pass through two metamorphic positions to transform among four states like the origami. As shown in Fig. 3, to connect the module 1 and module 0 , it needs to connect the revolute joints $\mathrm{R}_{15}$ with $\mathrm{R}_{07}$ and revolute joints $\mathrm{R}_{16}$ with $\mathrm{R}_{08}$. If these two modules are completely folded, the axes of these four revolute will be collinear, so that the module 1 can realize a rotation around this revolute axis relative to module 0. Similarly, module 1 connects with module 2 by connecting $R_{17}$ with $R_{25}$ and $\mathrm{R}_{18}$ with $\mathrm{R}_{26}$, respectively. Based on this method, the single limb of grasper that formed by four motion modules and one fixed module is assembled, as shown in Fig. 4(a).

As shown in Fig. 4(a), there are three motions from state 1 to state 4 for the single limb, including deployment motion 1 , deployment motion 2 and grasping motion. The corresponding topology of these three motions for the single limb is shown in Fig. 4(b), in this limb, modules are numbered from 0 to 4 and the number of facets in each module is 4 , so $i=0, \ldots, 4$ and $j=1, \ldots, 4$ in this figure, the establishment of the coordinate systems is beneficial to clearly understand the directions of the motions in this limb. In deployment motion 1, all facets in the same module can be considered as the same moving link, according to (3), this folded single limb which has the motions with 4 DOFs; when the limb in state 2 , which reaches the first metamorphic position, the facets in the same relative position for different modules can be considered as the same moving link, they form a planar $4 \mathrm{R}$ mechanism like Fig. 2, so there is only one DOF in deployment motion 2; in state 3 , the grasping facet is completely deployed, the two revolute joints between each module are collinear again, where is the second metamorphic position, and all facets in the same module
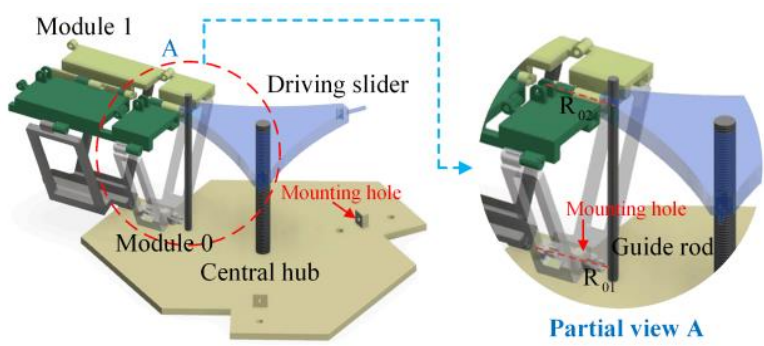

Figure 5. Assembly of single limb and central hub

reconstitute the same moving link, so there are also 4 DOFs in grasping motion, but the directions of revolute joints are different with deployment motion 1 . According to the analysis, this single limb can change the number of moving links by coaxial metamorphic method to realize the three motions, and there are two metamorphic positions of such single limb.

\section{B. Assembly of the 3-Limb Grasper}

In some applications, it can take only one limb to realize the grasping motion, especially for regular cylindrical objects. However, for the large-scale and non-cooperative objects, it is required to increase the number of limbs to grasp the objects in three-dimension. We have known that if the three limbs of grasper are distributed in center symmetry, it can grasp the 3D objects steadily. Thus, we need assemble the three proposed single limbs to form a 3-limb ODMGR.

According to the motion characteristics of single limb, we can know that the positions of the four modules in this limb have changed after three motions, and we can not find a fixed mounting position, so that the fixed module 0 is used to connect the central hub. Detailed assembly is as shown in Fig. 5. Since the function of module 0 is to connect the motion modules to central hub, and it also need to realize the deployment motion 2 , there is only the position of $R_{01}$ will not change in all states. There are three mounting holes on the central hub, which are used to connect the $\mathrm{R}_{01}$ of three limbs, respectively. The driving slider is mounted on the drive screw at center of hub, and its position is limited by the guide rod in the direction of the three limbs. Meanwhile, the driving slider connect with module 0 at $\mathrm{R}_{02}$. Therefore, the driving slider can be driven to let the $\mathrm{R}_{02}$ move along the guide rod so as to realize the deployment motion 2 in each limb.

Similar with the single limb, the all 3 limbs of grasper can be assembled on the central hub in center symmetry, as shown in Fig. 6. Then, the driving methods to obtain the four states of this grasper can be designed:

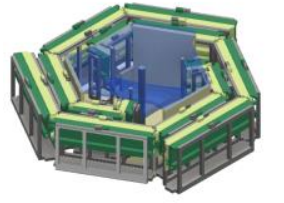

Stored state

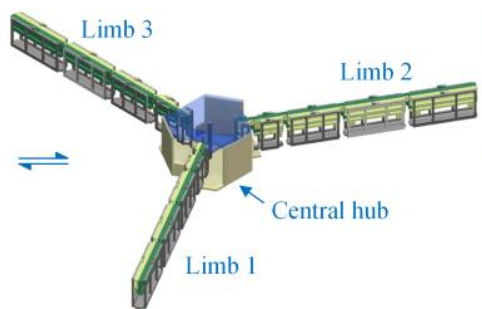

First deployed State

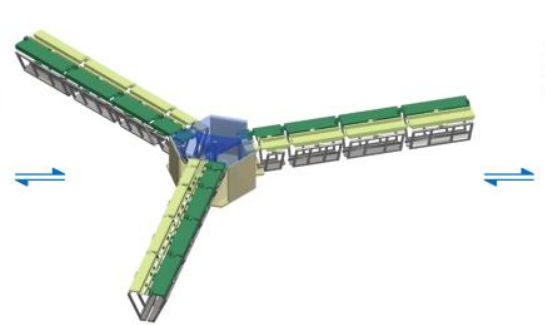

Grasping facet deployed state

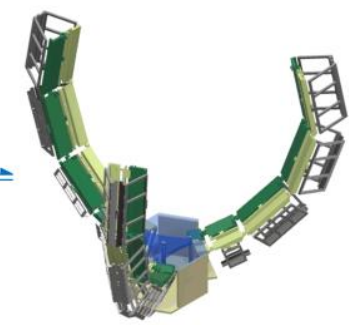

Grasping state

Figure 6. The ODMGR with three limbs 
State 1: Each limb is tightly stored around the central hub by a rope-driven method that controlled by a single motor to achieve the underactuated stored.

State 2: Control the driving rope relaxes slowly to ensure each limb can achieve the deployment motion 1 by the restoring force of the torsional springs that installed at the connection positions between the modules.

State 3: Use the single motor to control the motion of driving slider assembled at the central hub, then the three limbs that connected with driving slider can achieve the deployment motion 2 synchronously.

State 4: Each limb is controlled by a single motor using rope drive method to achieve the adaptive grasping.

\section{THE DEPLOY/FOLD RATIO AND KINEMATIC ANALYSIS}

\section{A. The Deploy/Fold Ratio Analysis}

In the special application of robotic grasper, especially in space engineering, it is required to increase the number of basic modules so that the grasper can be used to grasp the large-scale unknown objects, and it is a key problem that to improve the deploy/fold ratio. The folded and deployed configurations of a limb with four motion modules and a fixed module are as shown in Fig 7, where $a$ is the side length of the hexagonal central hub that the robotic grasper stored around; $l_{i}$ is the length of the $i$ th module; $d$ is the thickness of the folded state of each module; in a limb, $L_{1}$ and $L_{2}$ are the maximum lengths between the end module and center of hub in folded and deployed configurations, respectively.

The geometric relationships of ODMRG are as shown in Fig. 7(a), and the auxiliary shapes with different colors are used to represent the length relations of each module. the length of each module depends on $b$, which can be calculated as

$$
b=\frac{a}{\cos \alpha}
$$

thus, the length of each module can be given as

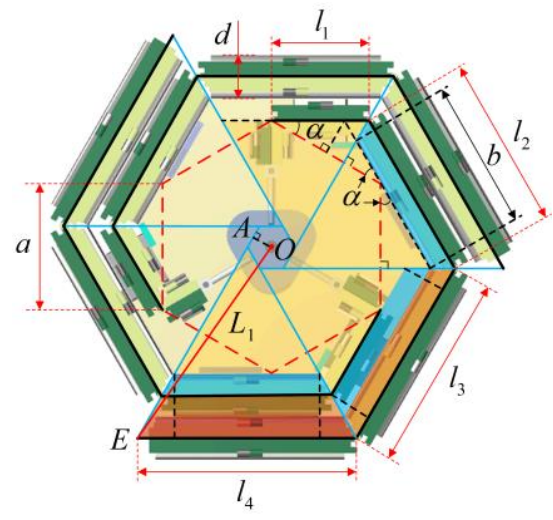

(a) Length of limb in folded configuration

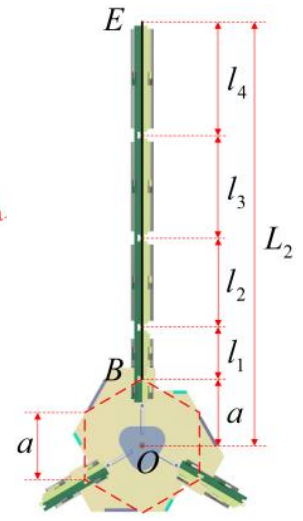

(b) Length of limb in deployed configuration
Figure 7. Deploy/fold ratio analysed of a limb

$$
\left\{\begin{array}{l}
l_{1}=\frac{a}{2 \cos \alpha}+d \tan \alpha \\
l_{2}=b+d \tan \alpha \\
l_{3}=b+2 d \tan \alpha \\
l_{4}=b+3 d \tan \alpha
\end{array}\right.
$$

Substitute (4) into (5), we can summarize

$$
l_{i}= \begin{cases}\frac{a}{2 \cos \alpha}+d \tan \alpha & i=1 \\ \frac{a}{\cos \alpha}+(i-1) d \tan \alpha & i=2,3,4 \ldots\end{cases}
$$

where the angle $\alpha$ is constant in this configuration, i.e. $\frac{\pi}{6}$.

Based on the special shape of folded configuration, the maximum stored length of each limb in folded state is

$$
L_{1}=\sqrt{\mathbf{O} \mathbf{A}^{2}+\mathbf{A} \mathbf{E}^{2}}=\sqrt{\left(\frac{d}{2}\right)^{2}+\left(\frac{d}{2} \tan \alpha+l_{n}\right)^{2}}
$$

where $l_{n}$ is the length of the end module.

According to (6), the length of each limb can be summarized as

$$
L=\sum_{i=1}^{n} l_{i}=\frac{(2 n-1) a}{2 \cos \alpha}+\frac{\left(n^{2}-n+2\right)}{2} d \tan \alpha
$$

where $n$ is the number of modules. Then, the maximum length of each limb in deployed configuration is as shown in Fig. 7(b) and can be given as

$$
L_{2}=L+\mathbf{O B}
$$

Substitute (7), (8) and (9), the deploy/fold ratio $\rho$ of such limb with $n$ motion modules can be easily obtained as

$$
\rho=\frac{L_{2}}{L_{1}}=\frac{\frac{(2 n-1)+2 \cos \alpha}{2 \cos \alpha} a+\frac{\left(n^{2}-n+2\right)}{2} d \tan \alpha}{\sqrt{\left(\frac{d}{2}\right)^{2}+\left(\frac{d}{2} \tan \alpha+l_{n}\right)^{2}}}
$$

For this ODMRG, one conclusion can be drawn that the length of each module is determined by the side length of the hexagonal central hub and the thickness of the module in folded state, i.e., $a$ and $d$, which influences the stiffness of the grasper. If these two parameters are determined, we can easily obtain the length of each module. And since the angle 


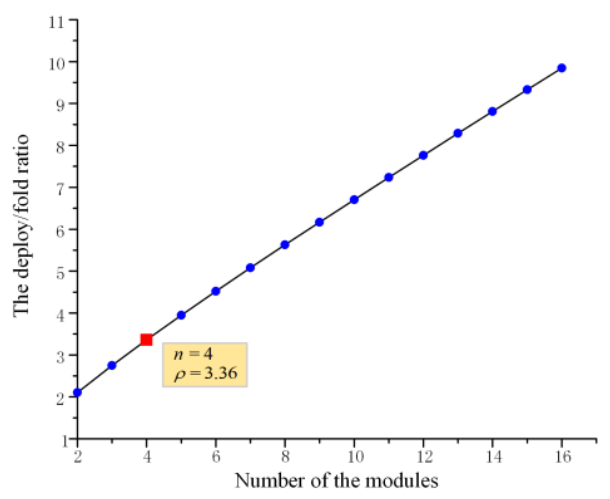

Figure 8. Deploy/fold ratio related with the number of modules

$\alpha$ is constant, the deploy/fold ratio is mainly determined the values of $a, d$ and $n$. In this paper, the values of $a$ and $d$ are $83 \mathrm{~mm}$ and $34 \mathrm{~mm}$, respectively, and the number of modules is 4 , the real deploy/fold ratio of this grasper is 3.36 .

In addition, to grasp the large-scale unknown objects, we can increase the number of modules. When other design parameters are the same, the deploy/fold ratio of the grasper increases as the number of modules adding, as shown in Fig. 8, and this ratio increases almost linearly. This characteristic is the greatest advantage of the ODMRG, which means that the length of the grasper can increase a lot, but the stored volume increases a little, so it is suitable for space application.

\section{B. Workspace and Deterity Analysis}

As to grasp the large-scale objects with different shapes, it is necessary to analyze the workspace of the proposed grasper. Since the fixed module of the single limb is not moving in grasping state, $\theta_{i}$ presents the grasping angle for the other four motion modules, where $i=1 . . .4$.

For single limb of grasper, if the motion module 4 is 90 degrees from the fixed module in the grasping position, the single limb will envelop at least a quarter of the grasping object, and if the angle between motion module 4 and fixed module is greater than 270 degrees, the form-closure for grasping object will be formed. Then, because there are three limbs are used to grasp together, the grasping angles for all motion modules can be limited

$$
\frac{\pi}{2} \leq \theta_{1}+\theta_{2}+\theta_{3}+\theta_{4} \leq \frac{3 \pi}{2}
$$

For each grasping angle, we limit it in $\left[0^{\circ}, 67.5^{\circ}\right]$ by structural design. Then, the workspace of the single limb is shown in Fig. 9.

The workspace of proposed grasper with three limbs is shown in Fig. 10. We can see that the range of grasping of proposed 3-limbs ODMRG is relatively large, at the same time, since the three limbs are centrosymmetric distributed, it is possible to be used to grasp some non-cooperative objects.

Furthermore, the dexterity of grasper determines the ability of the grasper position and orient end-effector, which is also

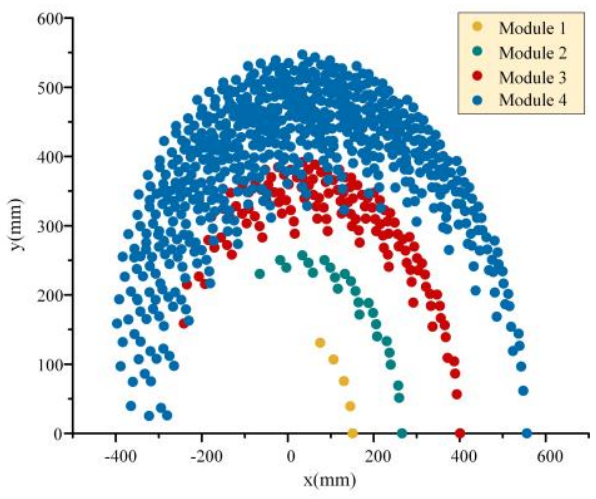

Figure 9. Workspace of a single limb

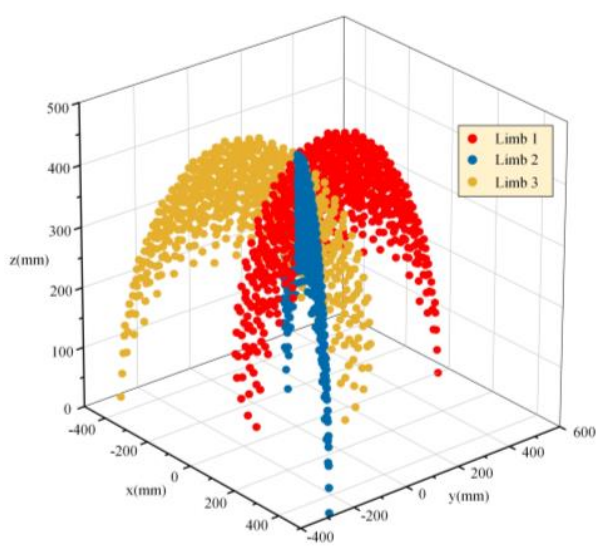

Figure 10. Workspace of the grasper consisting of three limbs

necessary to evaluate the reasonability of grasper. Since it is independent for each limb to drive them, we just need to analyze the dexterity of single limb. To raise the dexterity, we can let the grasping angles of the modules to be same, i.e., $\theta_{1}=\theta_{2}=\theta_{3}=\theta_{4}=\theta$. Then, the dexterity of the proposed grasper can be calculated follows:

$$
w=\sqrt{\operatorname{det}\left|\boldsymbol{J} \cdot \boldsymbol{J}^{T}\right|}
$$

where the $\boldsymbol{J}$ is the Jacobian matrix of the grasper. Based on these calculations, we can get that the dexterity is highest when $\theta=45^{\circ}$ in the finite range for grasping angle.

\section{PROTOTYPE AND TEST}

To demonstrate the feasibility of the proposed grasper, a physical prototype is manufactured. As shown in Fig. 11, the 3limbs ODMRG can be tightly stored around the central hub in stored state; then, the first metamorphic position in the state 2 is reached by the deployment motion 1; by the deployment motion 2, it can translate to state 3 from state 2 and can reach the second metamorphic position; finally, in grasping state, it can be used to grasp the target objects by grasping motion. This prototype not only verifies the feasibility of the designing scheme, but also proves that the ODMRG has excellent stored efficiency. 


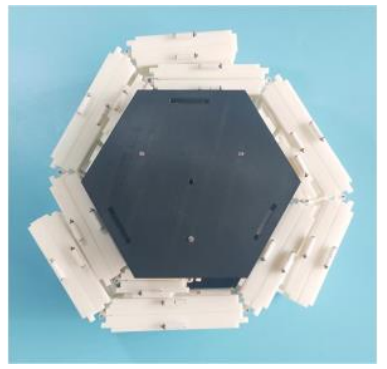

(a) Stored state

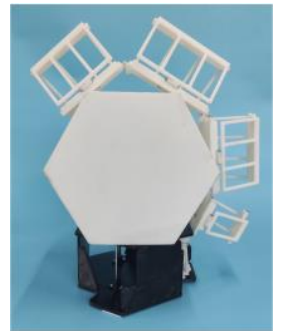

(a) Hexagonal prism object grasping

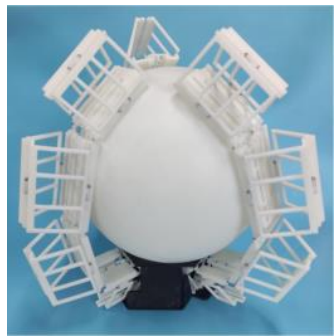

(c) Small size spherical object grasping

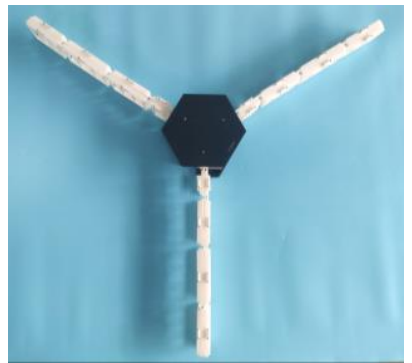

(b) First deployed state

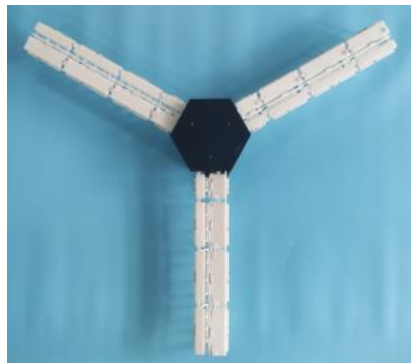

(c) Grasping facet deployed state

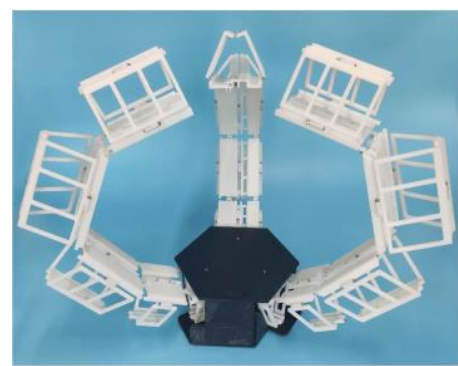

(d) Grasping state

Figure 11. The physical prototype of ODMRG in four states

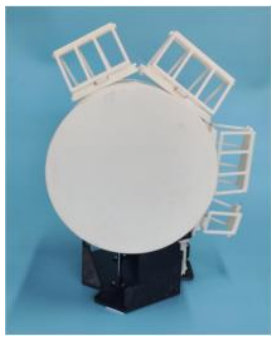

(b) Cylindrical object grasping

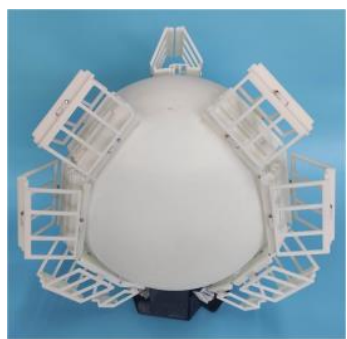

(d) Big size spherical object grasping

Figure 12. Shape adaptability during the grasping motion

To show the shape adaptability of proposed grasper, the grasping of different shape and size objects have been demonstrated. As shown in Fig. 12, for some column shape objects, such as hexagonal prism and cylinder, they can be grasped only need a single limb of grasper, which are shown in Fig. 12(a) and (b); for some other three-dimensional objects, three limbs of grasper are needed work together to grasp these objects, different size of ball-shape objects are grasped as shown in Fig. 12(c) and (d), which also prove that the proposed ODMRG has good shape and scale adaptability.

\section{CONCLUSION}

This paper proposed an origami-inspired deployable metamorphic robotic grasper (ODMRG), which could be stored tightly in the stowed configuration and had the grasping mobility to grasp the unknown objects in grasping configuration. The mobility of such grasper was analyzed during the deployment motion 1, the deployment motion 2 , and the grasping motion, respectively, which indicated that such grasper need pass through two metamorphic positions from stored state to grasping state. In addition, the derived equations shown that the deploy/fold ratio of this type of grasper can be

increased as the number of modules in each limb adds. Kinematic analysis illustrated that the proposed grasper had a large reachable workspace and great dexterity. Finally, a physical prototype was manufactured to show that such grasper not only can realize the desired motions, but also has the adaptability to grasp different shaped objects.

\section{REFERENCES}

[1] R. Deimel, and O. Brock, "A novel type of compliant and underactuated robotic hand for dexterous grasping," The International Journal of Robotics Research, vol. 35, pp. 161-185, August 2015.

[2] J. P. Grotzinger, J. Crisp, et al. "Mars Science Laboratory Mission and Science Investigation," Space Sci Rev, vol. 170, pp. 5-56, September 2012

[3] I. Loomis, "Space science. Air Force turns a keen eye on space junk," Science, vol. 347(6218), p. 115, January 2015.

[4] K. Tai, A. R. E. Sayed, M. Shahriari, M. Biglarbegian, and S. Mahmud, "State of the Art Robotic Grippers and Applications," Robotics, vol. 5(2), p. 11, June 2016.

[5] R. Ozawa, and K. Tahara, "Grasp and Dexterous Manipulation of Multi Fingered Robotic Hands: A Review From a Control View Point," Advanced Robotics, vol. 31, pp. 1030-1050, October 2017.

[6] D. M. Gan, J. S. Dai, and Q. Z. Liao, "Constraint analysis on mobility change of a novel metamorphic parallel mechanism," Mechanism and Machine Theory, vol. 45(5), pp. 1864-1876, December 2010.

[7] C. Zhao, H. W. Guo, and R. Q. Liu, "Actuation distribution and workspace analysis of a novel 3(3RR1S) metamorphic serial-parallel manipulator for grasping space non-cooperative targets", Mechanism and Machine Theory, vol. 139, pp. 424-442, September 2019.

[8] C. Shi, H. W. Guo, M. Li, et al, "Type synthesis of deployable singleloop overconstrained linkages based on Bennett linkages," Mechanism and machine Theory, vol. 120, pp. 1-29, February 2018.

[9] G. T. Li, H. L. Huang, H. W. Guo, and B. Li, "Design, analysis and control of a novel deployable grasping manipulator," Mechanism and Machine Theory, vol. 138, pp. 182-204, August 2019.

[10] G. L. Jia, H. L. Huang, and B. Li, "Synthesis of a novel type of metamorphic mechanism module for large scale deployable grasping manipulators," Mechanism and Machine Theory, vol. 128, pp. 544-559, October 2018.

[11] S. J. Kim, D. Y. Lee, G. P. Jung, and K. J. Cho, "An origami-inspired, self-locking robotic arm that can be folded flat," Sci. Robotics, vol. 3(16), p. 2915, March 2018.

[12] S. A. Zirbel, R. J. Lang, M. W. Thomson, et al, "Accommodating thickness in origami-based deployable arrays," J. Mech. Des. Vol. 135(11), p. 111005, November 2013.

[13] T. G. Nelson, T. K. Zlmmerman, et al, "Developable Mechanisms on Developable Surfaces," Sci. Robotics, vol. 4, p. 3029, February 2019.

[14] C. D. Onal, R. J. Wood, and D. Rus, "An origami-inspired approach to worm robots," IEEE/ASME Trans. on Mechatronics, vol. 18(2), pp. 430438, April 2013. 